\title{
MULTI-DOMAIN DESIGN ASSESSMENT FOR AEROSPACE COMPONENTS INCLUDING WELD ACCESSIBILITY
}

\author{
Isaksson, Ola (1); \\ Kipouros, Timos (2); \\ Martinsson, Julian (1); \\ Panarotto, Massimo (1); \\ Kressin, Jonas (3); \\ Andersson, Petter (4); \\ Clarkson, John, P. (2) \\ 1: Chalmers university of technology; \\ 2: University of Cambridge; \\ 3: Fraunhofer-Chalmers Research Centre for Industrial Mathematics; \\ 4: GKN Aerospace Engines
}

\begin{abstract}
Aeroengine manufacturers need to better include assessment of risk and cost for realising the novel products needed to meet the ambitions sustainability driven targets for air transport. Radical technologies are needed that simultaneously require critical manufacturing processes to be assessed already in conceptual design.

In this paper, a multi-domain framework for conceptual design and evaluation is proposed that provide the ability to interactively explore the concepts that simultaneously allow a wider range of architectures can be assessed and still include weldability of the concepts.

It has been demonstrated how high level, and function driven conceptual design alternatives can be modelled and evaluated to analyse risk and resilience of architectures. Geometrical concepts generated for the most interesting regimes using design of experiments covering a desired design space. For each CAD-model the welding process can be simulated to assess feasibility and lead time for welding, and return quantified results to be included in an integrated results data set for interactive decision making. The paper is the first report from a research project that improve concurrent design of product and production concepts.
\end{abstract}

Keywords: Design for X (DfX), Conceptual design, Multi- / Cross- / Trans-disciplinary processes, Early design phases

\section{Contact:}

Isaksson, Ola

Chalmers University of Technology

Product and Production Development

Sweden

ola.isaksson@chalmers.se

Cite this article: Isaksson, O., Kipouros, T., Martinsson, J., Panarotto, M., Kressin, J., Andersson, P., Clarkson, J. P. (2021) 'Multi-Domain Design Assessment for Aerospace Components Including Weld Accessibility', in Proceedings of the International Conference on Engineering Design (ICED21), Gothenburg, Sweden, 16-20 August 2021. DOI:10.1017/ pds. 2021.483 


\section{INTRODUCTION}

A green and sustainable future of air transport drives manufacturers to develop radically new solutions. In Europe, such demonstration programs are already well advanced (Clean sky, 2020), yet even more disruptive technologies are expected to be needed. From a business perspective, new architectures need to be carefully evaluated based on their risk and cost of realisation already in the conceptual design phase. One challenge is how to allow an expanded range of alternative product concepts while simultaneously assessing impact of critical manufacturing processes, something that requires geometrical models on a relatively refined level of detail and available first after concepts have been selected. As one example, the weld robots used for welding require spatial access to perform welding within a constrained angle and the decision to assemble a component using welding vs choosing a fully cast design is determined very early in design. Geometrical CAD models are also needed for engineering analysis for mechanical, thermal and fluid flow performance.

This paper is a first report from a project seeking to integrate manufacturability in digital experiments for conceptual design, making use of design automation to generate geometry in combination with digital experiments that include manufacturing equipment and simulate weld processes. Designers need to interactively assess the associated risk and cost of manufacture for alternative concepts and trade other performance factors with cost and realisation performance. The idea is to investigate how the design space can be defined based on expected high level functionality, and how known relations and dependencies can be represented for such architectures to gain knowledge of their behaviour. Based on this pre-embodiment study geometry is generated such that design of experiments can be conducted through simulation. In particular, simulation-based methods that can assess weld accessibility have not been used in conceptual design experiments to date. In addition, using data and visual analytics approaches, the ability of the designers is further extended to interactively assess what architectures are most viable and resilient at this stage of the process.

\section{BACKGROUND}

The present work requires the integration and extension of several state of the art design related modelling and simulation capabilities following Model Based Systems Engineering (MBSE) principles Pagliuca et al. (2019). Hence a brief literature review on modelling based design methodology and simulation for manufacturability in design is appended. Recently, multidisciplinary design systems have been reported from a UK-based research project, APROCONE (Piotrowski et al., 2019), representing state of the art technologies for interactive decision making for design experiments. To advance the assessment of manufacturability there is a need to replace, or expand, the predominately qualitative practices in Design for Manufacturing (DFM) with simulation based and quantified methods, such as proposed by Söderberg et al. (2018).

\subsection{Early architecture design and analysis}

The term "product architecture" is often used to describe how physical components, technologies and solutions accomplish desired functions. The design of a product architecture distinguishes two processes: architecture definition and architecture analysis. To support the definition of an architecture, function modelling is widely adopted in industry, and its benefits have been recognised (Eisenbart et al., 2017). Popular techniques for functional modelling are the Function Block Diagram (FBD) and the Function Analysis System Technique (FAST). However, a limitation of these approaches is that they do not allow the representation of design solutions and functions in the same model. Furthermore, these techniques do not allow to represent architectural innovations where the functions and the solutions remain the same, but where linkages are changed. For these reasons novel approaches of conducting function modelling have been proposed. For example, Enhanced Function-Means (EF-M) modelling (Müller et al., 2019) enables the representation of both functions, solutions and interactions in the same model. The analysis of the defined architecture often focuses on analysing the degree of coupling or modularity (Otto et al., 2013). This is because at this stage no detailed model of the architecture is available. Therefore, analysis methods based on Design Structure Matrices (DSMs) are helpful and effective at this stage, such as the Change Propagation Method (CPM) (Clarkson et al., 2004). In this context, the EF-M method also supports the extraction of a DSM from the function model, which can be used for architecture analysis. 
One limitation of function models and DSMs, independently from the techniques used, is that they do not allow to assess product dimensions (e.g. weight, drag) that would require representations with a higher degree of maturity (e.g., CAD) from which simulations (e.g. FEM) can be run. Recent research (Müller et al., 2020) has focused on connecting function models with geometry models, extending current Design Automation (DA) and (KBE) techniques. The next section focuses on reviewing such techniques.

\subsection{Geometry concept variation modelling}

Traditionally, flexible 3D-models in Computer Aided Design (CAD) has been conceived through highlevel parameterisation such that a selection of dimensions can be varied by editing a few design parameters (Straßer and Seidel, 1989). This is supported by most history-based CAD software used in industry, as it enables reuse of components, as well as model flexibility such that a range of similar products can be conceived using one CAD-model. However, while it does enable a degree of variability that is enough to make minor model changes, variation on a larger scale is hard to achieve by merely varying high-level CAD parameters.

In the field of Knowledge Based Engineering (KBE), design automation is often achieved by leveraging parameterisation and programming. Engineers can create applications that generate designs based on rules and classification, enabling the reuse of engineering knowledge (Verhagen et al., 2012). An example of this can be found in Amadori et al. (2012), where programming techniques and parametric CAD-models are used in conjunction to create highly flexible 3D models. This enables design engineers to quickly instantiate a 3D-model which either can be further built upon, or used as is.

\subsection{Manufacturability modelling and simulation}

Today, it is possible to simulate manufacturability once a geometrical representation exists, and Landahl et al. (2016) showed how weld paths can be simulated to guide designers where to split the assembly. Methodologies such as Design for Manufacture/Assembly (DFMA) and Six Sigma can also be implemented within the simulated environment. For example, new software tools have been emerged in the recent years for automatic collision free robot path planning for assemblies. A large majority of these applications have been focused on collision free planning of robot welding. The result shows a 25 percent improvement in cycle time for a real stud welding line with 10 robots (Segeborn et al., 2013). Despite these advances, the needs of the aerospace industry require novel DFMA approaches and the use of advanced manufacturing models. In the context of welded aircraft components, much of the welding experimentation relies on physical testing, which means that welding producibility aspects are considered after the design has already been established. In addition, welding optimisation research mainly focuses on welding process parameters, overlooking the potential impact of product design (Madrid et al., 2019), as well as of variations during manufacturing from nominal values (e.g., tolerances). As a consequence, redesign loops and welding rework increases product cost.

There is a need to to introduce the ability to assess manufacturability into a multidisciplinary design optimisation cycle to facilitate interactive trade-off and optimisation between product design and manufacturing process alternatives in a digital and interactive environment.

\section{MULTI-DOMAIN DESIGN ASSESSMENT METHODOLOGY}

The proposed methodology (Figure 1) benefits from existing infrastructure of multidisciplinary tools for structural engine components used by the industrial partner. The current practice at the industrial partner is shown in the upper part of (Figure 1) where Design of Experiments (DoEs) are performed in a parametric design space and the corresponding CAD geometries are automatically defined. Physics-based simulations produce the critical performance analysis that are necessary in order to evaluate the designs. There is currently a gap in including manufacturing and welding simulations in the early design phases, as well as simulation of non-geometrical properties (e.g. project risk, development process efficiency). In the lower part of the figure, the novel approach introduced by this research is shown. The approach starts with capturing and considering the desirable new functionality and new technologies of the engine component in an EF-M model. This model generates automatically a series of a Design Structure Matrix as (DSMs) which represent all conceptual combinations of design concepts. A change propagation analysis is performed thereafter. These analyses effectively performs a quantified risk assessment and a cost 


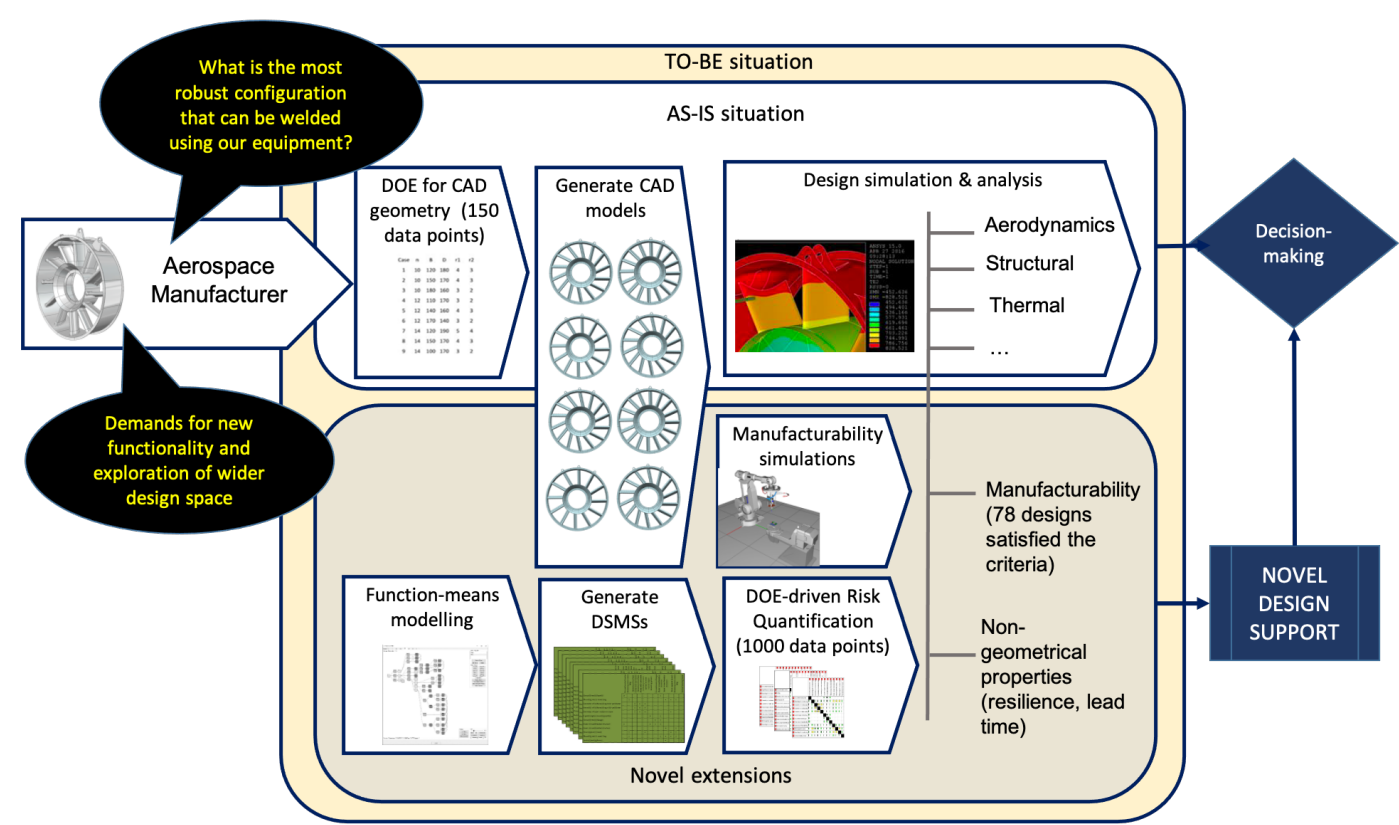

Figure 1. Overall process for multi-domain design assessment of structural engine components.

of realisation estimation. Such models can be used in conjunction with the parametric CAD models, and hence, new DoEs can be instructed. Additional state-of-the-art manufacturing process models and tools enrich and expand the diversity of the dataset, but produce the necessary information that characterise the accessibility of direct deposition additive manufacturing tools, weld tools and inspection processes. The last step of the process is to define and express the relationships between the different evaluation criteria in a synthetic multi-dimensional design space, where physics-based dimensions (e.g. aerodynamics) are combined with manufacturing criteria and non-geometrical properties (e.g. project risk) for interactive decision-making. Techniques such as enhanced Parallel Coordinates can be used to allows for an in-depth analysis of the multi-dimensional data and directly supports the decision-making process.

\section{PRELIMINARY RESULTS}

To illustrate the application of the proposed methodology, an industrial case is presented. A turbine rear structure (TRS) (see Figure 2), is an aerospace component located in the rear end of a jet engine. The TRS has a range of functional criteria from various fields of engineering. They need to be able to withstand significant thermal and structural loads. In addition, to optimise fuel efficiency, they need to be as light and aerodynamic as possible. These functionality criteria must be balanced in order to obtain an optimal design. At the same time, manufacturability constraints need to be taken into account and need to be traded against functional requirements. In this paper, manufacturing requirements are treated as non-functional requirements and are independent from how the functionality of final product is realised. However, they are evaluated so that they restrict the design space.

\subsection{Digital modelling of product architectures}

Previous studies on architectural modelling (e.g. Jiao et al. (2007)) have focused on modular products where there is a clear separation between the physical components. Also, in modular products it is rather intuitive to associate each component to its underlying function. The TRS represents instead the case of a highly integrated product, meaning that all required functions are satisfied by a single, monolithic component Raja et al. (2019). Therefore, EF-M modelling has been used in order to separate the elements of the system in order to identify which elements of this single monolithic component contribute to satisfy the product's functions. Figure 2 shows how the EF-M technique has been used to represent functions, solutions and interactions between them.

For example, the upper left part of Figure 2 show how the outer structure of the TRS fulfils two functions: 'Contain failed turbine parts' (solved by using the outer structure as a barrier) and 'Maintain 
structural Integrity' (solved with the actual outer structure). These two solutions presents many interactions with other parts of the TRS (shown as red arcs in Figure 2. For example, a change in the diameter of the outer structure implies a change in the vane length (the grey parts in the TRS CAD model in Figure 2). However, the vane is not directly attached to the outer structure. This 'attach' function is solved in this TRS by the use of 'mounting stumps' (the green parts in the TRS CAD model in Figure 2) in order to facilitate the vane attachment through welding. the EF-M model can support the representation of secondary effects between design solutions. For example, changing the number of vanes and the lean angles will change the number and the geometry of the mounting stumps, which on their turn impact the performances of the outer circumferential structure (and its ability to act as a 'barrier' to contain failed parts). At the same time, these changes impact the welding time and welding performances. The results from the EF-M model ban be represented in a DSM (lower right part of Figure 2). This can allow to analyse the interactions between these design solutions already with such first order representations.



Figure 2. EF-M modelling of TRS, and extraction of associated DSM. FR = functional requirement, $D S=$ design solution.

As soon as the DSM model is available, we can introduce likelihood and impact values of a 'change' for each connection in the model. The meaning of 'change' depends on the context, but typically we mean a change to the component due to a failure or change to the specifications. What is interesting though, is that a risk and behavioural analysis of the architecture is possible regardless the context at this stage of the process. Furthermore, the values of likelihood and impact might not be known at this stage. However, the Change Propagation simulation can produce a description of the generic behaviour of the system, or the TRS architecture in our example, and also reveal implicit relationships between components which represent high, or sometimes even higher risk than direct connections. 


\subsection{Design of Experiments-driven architecture simulations and risk quantification}

For illustrative purposes, we have analysed a reference architecture of the TRS. We consider the cause and effect relationship for each connection between two elements of the DSM shown in Figure 2, hence, this produces 30 connections. We then consider the likelihood and impact value of each connection as free variables in order to produce variations of this specific architecture. This results in 60 variables and a DoE based on the Latin Hypercude method has been setup and executed with 1,000 data points. With this CPM simulations-driven DoE we aim to introduce a mechanism to quantify the risk of an architecture and by identifying the most influential risk propagator, or the most susceptible receiver we can perform a first screening of the architecture components behaviour. In order to assess these architectural variations, we introduced two measures, namely Resilience and LeadTime. These two metrics are quantified based on the properties calculated in CPM simulations and the corresponding equations are shown in Figure 3. The definitions of these quantified risk properties are presented in Keller et al. (2009).

- Number of changed elements (RES1)

- Aggregated propagation of change (RES2)

- Resilience $=1 /($ RES1 + RES2)



- Number of propagation multipliers (LT1)

- Number of high in/out risk components (LT2)

- $\quad$ LeadTime $=1 /(\mathrm{LT} 1+\mathrm{LT} 2)$

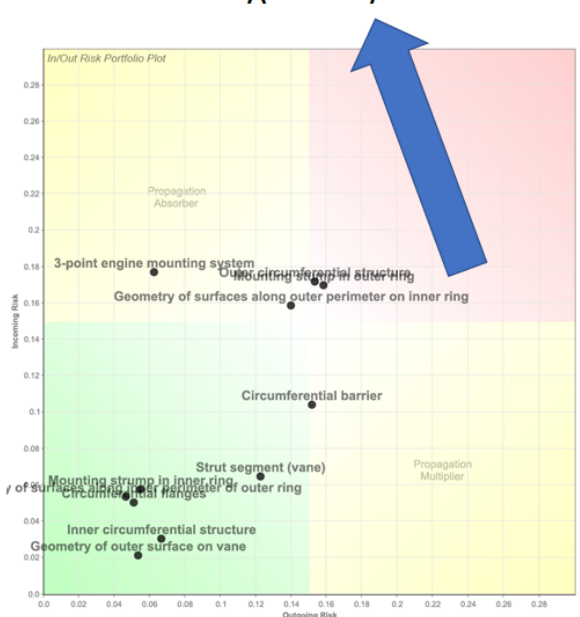

Figure 3. The output combined risk matrix (left) and the risk portfolio plot (right) from a Change Propagation simulation. The definitions of Resilience and Lead Time are also shown.

The Resilience is estimated by considering the number of affected components, so the less yellow boxes and the smaller the number of boxes that appear in the output combined risk matrix the higher the resilience. For the quantification of LeadTime we consider the number of propagation multipliers and the components that are highly linked, and hence, can potentially express a longer design team of these particular components with a negative impart to the overall lead time.

\subsection{Geometry-driven digital experiments}

The reference TRS CAD-model has been constructed such that it has several high-level parameters that can be varied (see Table 1). This enables a narrow design space exploration. To generate a batch of TRS models an algorithm was used that implemented a Latin hypercube sampling strategy. This ensured that all parameters had their entire ranges represented (see Table 1). The Latin Hypercube design was initialised such that $N=150$ and $K=8$, where $N$ is the amount of strata, and $K$ is the number of variables. A random value was then picked for each variable within each stratum, with exception of the vane count which was rounded up towards the closest integer. One example of the reference TRS can be seen in Figure 2.

\subsection{Welding modelling and simulation}

The generated CAD models were used to evaluate manufacturability of the TRS models through the software Industrial Path Solutions (Mark et al., 2014) and pre-modelled CAD models of the welding equipment were used. As base model for the evaluation a robotised welding cell was used, see Figure 4. 
Table 1. Reference model parameter variability

\begin{tabular}{||lrr||}
\hline CAD Parameter & Range & Unit \\
\hline \hline Vane count & {$[12,16]$} & N/A \\
Vane lean & {$[5,25]$} & {$\left[{ }^{\circ}\right]$} \\
Vane thickness & {$[1.5,3.5]$} & {$[\mathrm{mm}]$} \\
Vane upper weld line distance from outer ring & {$[25,60]$} & {$[\mathrm{mm}]$} \\
Outer-ring diameter & {$[920,1000]$} & {$[\mathrm{mm}]$} \\
Outer-ring thickness & {$[3,5]$} & {$[\mathrm{mm}]$} \\
Core diameter & {$[420,450]$} & {$[\mathrm{mm}]$} \\
Core thickness & {$[3,5]$} & {$[\mathrm{mm}]$} \\
\hline
\end{tabular}

The input to the manufacturability evaluation were also the piecewise linear curves for the weld joints. Based on this data, the following steps were adopted to evaluate manufacturability:



(a)

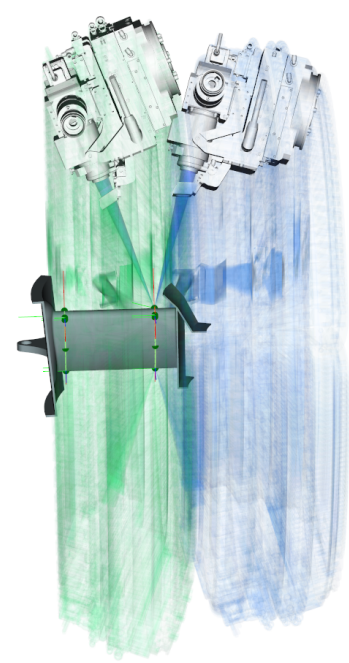

(b)

Figure 4. Manufacturability analysis for a TRS model.

1. Load model and weld curves into IPS.

2. Create a Task Definition from the input curves. The Task Definition is created based on methods described in (Mark et al., 2014), such that:

(a) The number of via points is minimised.

(b) The task definition is within a defined tolerance of $1 \mathrm{~mm}$ from the input curve.

(c) The $\mathrm{x}$-axis follows the curve tangent.

(d) The z-axis is defined in the welding direction.

(e) The velocity along the curve is set to $10 \mathrm{~mm} / \mathrm{s}$.

3. Sort out the relevant geometries included in each sub assembly step, see Figure 4a.

4. Sweep a welding tool along the Task Definition with varying tilt angle to find minimum and maximum angle without collision, see Figure $4 b$.

To test the manufacturability simulation, 150 TRS models were evaluated. For all models it was possible to follow the inner split line (right weld curve in Figure 4b) with both positive and negative tilt angles along the surface normal without collision. For the outer split line it was only possible to follow 78 of the 150 models with both positive and negative tilt angles. If we assume that a $0^{\circ}$ tilt angle is preferred to produce a high quality weld, we can then classify these 78 models as having a low level of weld accessibility. These results can be included in the multi domain evaluation and represented in the interactive decision-making as shown in Figure 5. 


\subsection{Interactive decision-making}

We perform multidimensional data visualisation and interactive decision-making analysis on a dataset that represents the variation of the likelihood and impact values of each connection in the DSM shown in Figure 2. After the DoE of 1,000 data points, as presented in Section 4.2, was performed the analysis showed that specific ranges of variability of likelihood and impact of very few connections expressed improved behaviour in terms of Resilience and LeadTime. Following this, an optimisation study was performed considering these ranges of variability. In Figure 5 it is shown a snapshot of the interactive analysis, where the blue polygon in Figure $5 \mathrm{~b}$ represents the solutions in the proximity of the Pareto front, and the interval selection of two connections shown in Figure 5a revealed a pattern. This means that there is a consistent behaviour within this reference architecture which expresses improved Resilience and LeadTime. It is notable that many of the likelihood and impact values are in the higher range of variability, which might not be expected. Indeed though, there are quite a few connections which take values in the lowest range of variability. With more detailed analysis, we expect to identify the components in the architecture that should be refined very early in the development process, but also the components which can be finely tuned or altered in a later stage without affecting the overall optimum behaviour of the product architecture. Even though the improved characteristics of the optimised design are clear, the three most influential components remain the same, as also shown in Figure 2.

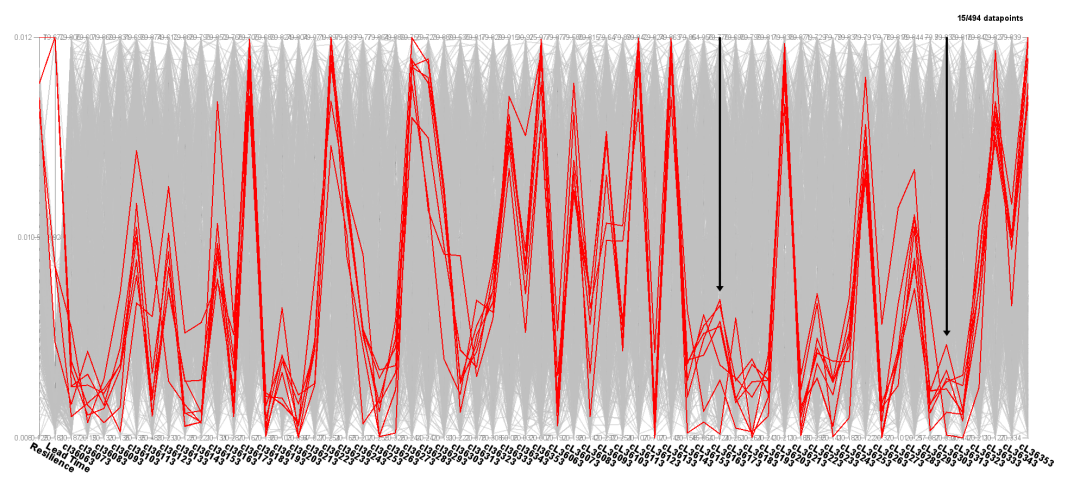

(a)

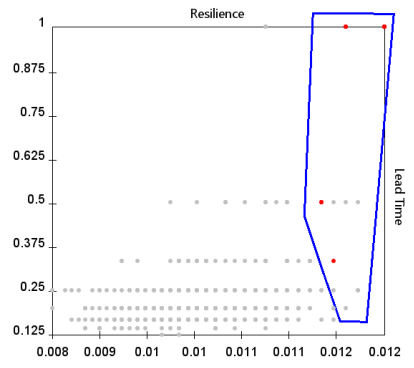

(b)

Figure 5. Visualisation of 62-dimensional design space in Parallel Coordinates, showing the likelihood and impact values for all 30 connections, and a simultaneous selection of the trade-off between Resilience and Lead Time in the Scatter plot.

\section{CONCLUSIONS}

Manufacturability, such as accessability of welding equipment is difficult to assess without detailed geometry and therefore not routinely a part of conceptual design. The multi-domain framework presented now provides the ability to interactively explore the performance of alternative concepts also including weldability characteristics. It has been demonstrated how high-level, and function-driven conceptual design alternatives can be represented (EF-M) and evaluated (DSM and Change Propagation) to analyse risk and resilience of architectures. Geometrical variability can be generated using a DoE that covers a desired design space. For each CAD-model the welding process can be simulated to assess feasibility and lead time for welding, and return quantified results to be included in an integrated results data set for interactive decision making. For future work, besides a fully automated and seamless integration, the criteria for decision making based on the weldability simulations need to be quantified. Despite the fact that manufacturing simulations require detailed geometric information, the generative modelling approach in combination with design automation allows these factors to be derived and included also in the pre-embodiment study, which is based on EF-M and Change Propagation analyses.

\section{ACKNOWLEDGEMENTS}

The project leading to this paper has received funding from the Clean Sky 2 Joint Undertaking under the European Union's Horizon 2020 research and innovation programme. Grant agreement No 887174. 


\section{REFERENCES}

Amadori, K., Tarkian, M., Ölvander, J. and Krus, P. (2012), "Flexible and robust CAD models for design automation", Advanced Engineering Informatics, Vol. 26 No. 2, pp. 180-195.

Clarkson, P.J., Simons, C. and Eckert, C. (2004), "Predicting Change Propagation in Complex Design", Journal of Mechanical Design, Vol. 126 No. 5, pp. 788-797, 10.1115/1.1765117.

Clean sky (2020), "Sustainable and Green Engines (SAGE)", . https://www.cleansky.eu/sustainable-andgreen-engines-sage

Eisenbart, B., Gericke, K., Blessing, L.T. and McAloone, T.C. (2017), “A dsm-based framework for integrated function modelling: concept, application and evaluation”, Research in Engineering Design, Vol. 28 No. 1, pp. 25-51.

Jiao, J.R., Simpson, T.W. and Siddique, Z. (2007), "Product family design and platform-based product development: a state-of-the-art review”, Journal of Intelligent Manufacturing, Vol. 18 No. 1, pp. 5-29.

Keller, R., Eckert, C. and Clarkson, P.J. (2009), "Using an engineering change methodology to support conceptual design", Journal of Engineering Design, Vol. 20 No. 6, pp. 571-587.

Landahl, J., Levandowski, C., Johannesson, H., Söderberg, R., Wärmefjord, K., Carlson, J.S., Kressin, J., Isaksson, O. and Vallhagen, J. (2016), "Using product and manufacturing system platforms to generate producible product variants", Procedia CIRP, Vol. 44, pp. 61-66.

Madrid, J., Lorin, S., Söderberg, R., Hammersberg, P., Wärmefjord, K. and Lööf, J. (2019), “A virtual design of experiments method to evaluate the effect of design and welding parameters on weld quality in aerospace applications", Aerospace, Vol. 6 No. 6, p. 74.

Mark, A., Bohlin, R., Segerdahl, D., Edelvik, F. and Carlson, J.S. (2014), “Optimisation of robotised sealing stations in paint shops by process simulation and automatic path planning", International Journal of Manufacturing Research, Vol. 9 No. 1, pp. 4-26, 10.1504/IJMR.2014.059597.

Müller, J.R., Isaksson, O., Landahl, J., Raja, V., Panarotto, M., Levandowski, C. and Raudberget, D. (2019), "Enhanced function-means modeling supporting design space exploration", AI EDAM, Vol. 33 No. 4, pp. 502-516.

Müller, J.R., Panarotto, M. and Isaksson, O. (2020), "Design space exploration of a jet engine component using a combined object model for function and geometry", Aerospace, Vol. 7 No. 12, 10.3390/aerospace7120173.

Otto, K., Levandowski, C., Forslund, A., Johannesson, H. and Söderberg, R. (2013), "Uncertainty modeling to enable software development platforms that can automate complex mechanical systems design", Proceedings of the International Conference on Engineering Design, ICED, Vol. 9 DS75-09, pp. $247-256$.

Pagliuca, G., Kipouros, T. and Savill, M. (2019), "Surrogate modelling for wing planform multidisciplinary optimisation using model-based engineering”, International Journal of Aerospace Engineering, Vol. 2019.

Piotrowski, W., Kipouros, T. and Clarkson, P.J. (2019), "Enhanced interactive parallel coordinates using machine learning and uncertainty propagation for engineering design", in: 2019 15th International Conference on eScience (eScience), pp. 339-348, 10.1109/eScience.2019.00045.

Raja, V., Kokkolaras, M. and Isaksson, O. (2019), “A simulation-assisted complexity metric for design optimization of integrated architecture aero-engine structures", Structural and Multidisciplinary Optimization, Vol. 60 No. 1, pp. 287-300.

Söderberg, R., Wärmefjord, K., Madrid, J., Lorin, S., Forslund, A. and Lindkvist, L. (2018), “An information and simulation framework for increased quality in welded components", CIRP Annals, Vol. 67 No. 1, pp. $165-$ 168, https://doi.org/10.1016/j.cirp.2018.04.118.

Segeborn, J., Segerdahl, D., Ekstedt, F., Carlson, J.S., Andersson, M., Carlsson, A. and Söderberg, R. (2013), "An Industrially Validated Method for Weld Load Balancing in Multi Station Sheet Metal Assembly Lines”, Journal of Manufacturing Science and Engineering, Vol. 136 No. 1, 10.1115/1.4025393. 011002.

Straßer, W. and Seidel, H.P. (1989), Theory and Practice of Geometric Modeling, Springer Berlin Heidelberg, 10.1007/978-3-642-61542-9.

Verhagen, W.J., Bermell-Garcia, P., Van Dijk, R.E. and Curran, R. (2012), “A critical review of Knowledge-Based Engineering: An identification of research challenges”, Advanced Engineering Informatics, Vol. 26 No. 1, pp. 5-15, 10.1016/j.aei.2011.06.004. 


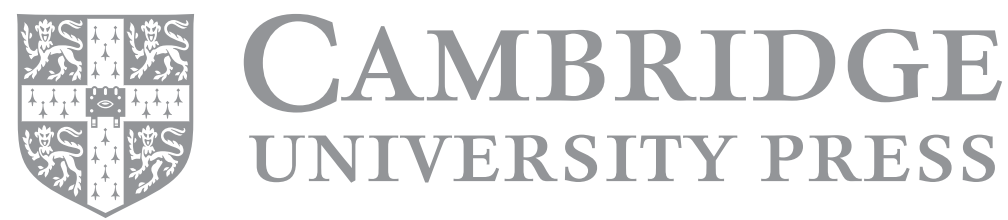

